\title{
Recent Advances in Intelligent Transportation Systems for Cloud-Enabled Smart Cities
}

\author{
Sang-Bing Tsai $\mathbb{D}^{1},{ }^{1}$ B. B. Gupta, ${ }^{2,3}$ Dharma P. Agrawal, ${ }^{4}$ Wenqing Wu ${ }^{(D)},{ }^{5}$ and Aijun Liu $\mathbb{D}^{6}$ \\ ${ }^{1}$ Regional Green Economy Development Research Center, School of Business, Wuyi University, Wuyishan 354300, China \\ ${ }^{2}$ National Institute of Technology, Kurukshetra 136119, Haryana, India \\ ${ }^{3}$ Asia University, Taichung, Taiwan \\ ${ }^{4}$ University of Cincinnati, Cincinnati, OH, USA \\ ${ }^{5}$ Tianjin University, Tianjin, China \\ ${ }^{6}$ Xidian University, Xi'an, China
}

Correspondence should be addressed to Sang-Bing Tsai; sangbing@hotmail.com and Wenqing Wu; wenqingw@tju.edu.cn

Received 28 July 2021; Accepted 28 July 2021; Published 15 August 2021

Copyright $(92021$ Sang-Bing Tsai et al. This is an open access article distributed under the Creative Commons Attribution License, which permits unrestricted use, distribution, and reproduction in any medium, provided the original work is properly cited.

The vision of a Smart City involves enriching the quality of life by gaining insights from data collected from interconnected sensors, devices, and people. Perpetual urban issues such as security, waste management, transportation, and traffic can be addressed by utilizing data to improve efficiency; however, to do this, all data needs to be stored in a location in which it can be easily accessed and used by all stakeholders, both private and governmental. The cloud service will help break down intergovernmental silos wherein different departments have no clear channel to communicate and understand data-based priorities of other departments-a factor seen as a major impediment to Smart City adoption. Security is also a major aspect of the new product, as the continued perpetuation of the "Internet of Things" has (and will) created demonstrable security concerns.

The concept of a Smart City focuses on cloud interoperability and connectivity scenarios, based on the Softwareas-a-Service (SaaS) delivery model. This approach holds the promise of reducing capital and infrastructure costs, while improving the efficiency of service provision within the Smart City framework. SaaS delivers software over the Internet, eliminating the need to install and run the application on private servers, simplifying maintenance, and enabling customers to use applications remotely through the IoT from anywhere in the world. Intelligent transportation systems are designed to support the Smart City vision, and the IoT can be applied both in intelligent transportation systems and in Smart Cities to form an advanced platform for novel applications; however, there are various issues and challenges that arise.

Therefore, this Special Issue mainly focuses on recent advances in intelligent and smart transportation systems for IoT-enabled Smart Cities, which addresses both the original algorithmic development and new applications. This collection spans a body of work that represents the efforts of 9 original research papers.

$\mathrm{X}$. Du et al. developed a sustainable GCU application system for intelligent transportation under blockchain. The results show that (1) solving social problems is the primary link, (2) economic tasks are mainly focused on smart contracts and affected by the social problems, (3) the continuous improvement of environmental issues requires a solution to social problems, and (4) the application system of blockchain in intelligent transportation needs to be built from three levels including the government layer, the company layer, and the user layer.

L.-L. Zhang et al. presented a traffic cyber physical system for urban road traffic signal control. With this proposed system, managers and researchers can realize the construction and simulation of various types of traffic scenarios, the rapid development, and optimization of new control strategies and can apply effective control strategies to actual traffic management. By carrying out a one-year practical test in Weifang City, Shandong Province, China, the system has been proved in terms of stability, security, scalability, practicability, and rapid practice, and verification of the new control strategy. 
In the context of smart finance, taking into account the economic conditions, social benefits, industry characteristics, and other indicators related to the measurement of supply chain financial risk, K. Wang et al. established a relatively comprehensive index system for the evaluation of supply chain financial risk, which provides a reliable reference for the objective completion of multiobjective measurements.

D. Zhao et al. combined the concept of blockchain with the shared bicycle system, and they creatively constructed a deposit management system for shared bicycles based on the blockchain technology. The results show that the outstanding advantages of the proposed deposit management mode, which include improving deposit supervision and guaranteeing user deposit security, are also conducted.

Y. Chen et al. analyzed the sustainable development capability of the port logistics system for outbreak emergency supplies from overseas and formulated response strategies and robust collaborative optimization methods. The optimized and robust system is obtained through formula derivation and analysis, which realizes the coordinated optimization of emergency logistics infrastructure positioning and emergency rescue vehicle path positioning and minimizes the economic loss caused by the outbreak. Research data show that the shortest path can be planned between each material supply location.

D. Xiao et al. used the BP neural network data fusion method based on the multisource data fusion method to build a model to monitor the slope construction of the expressway. This study used a multisource data fusion method to invert the soil parameters to establish a monitoring point-slope model, analyze and calculate the displacement values at different depths at the monitoring points, and compare them with the measured displacement values.

Considering that the charging behaviors of users of electric vehicles (EVs) (including charging time and charging location) are random and uncertain and that the disorderly charging of EVs brings new challenges to the power grid, X. Xu et al. proposed optimal electricity pricing strategy for EVs based on region division and time division.

$\mathrm{K}$. Lu and X. Wang built a concept model to investigate the effect of related factors on travelers' behavioral intentions of ride-hailing services adoption. In the concept model, the perceived value consists of two parts, i.e., perceived benefit and perceived sacrifice. Based on a survey in Nanjing, China, an empirical analysis was put forward to investigate the relationships between related factors and travelers' behavioral intention to adopt ride-hailing services. The results demonstrate that perceived value is positively related to behavioral intention. And, the factors of perceived benefit are related to perceived value positively, while the factors of perceived sacrifice have a negative effect on perceived value.

D. Li and X. Lin divided the high-speed rail industry chain into infrastructure construction market and manufacturing market of mobile equipment and used the empirical method of new experience industry organization to measure the market power premium of the high-speed rail upstream market. It is found in the study that the upstream market of the high-speed rail industry has a tendency of insufficient enterprise entry and the total social welfare increases with the increase in the number of upstream enterprise entries. Furthermore, the profit of enterprises in the upstream market of the high-speed rail decreases with the increase in the number of enterprises in the upstream.

In conclusion, we believe that this research topic presents a conceptual and practical broad work and that the papers included in this collection clearly contribute to our understanding of the recent advances of intelligent transportation systems for cloud-enabled Smart Cities.

\section{Conflicts of Interest}

The Guest Editors declare no conflicts of interest.

Sang-Bing Tsai

B. B. Gupta

Dharma P. Agrawal

Wenqing $W u$

Aijun Liu 